\title{
Análise da resistência de placas ósseas de acrílico fabricadas a laser quando submetidas a diferentes forças que agem sobre o foco de fratura
}

[Analysis of the resistance of laser-made acrylic bone plates when subjected to different forces acting on the fracture focus]

\author{
M.P.C. Xisto ${ }^{1}$, D. Barauna Júnior ${ }^{2}$, P.H.N. Cardoso ${ }^{1}$, N.C. Olivier $^{3}$, A.C. Antonelli2 ${ }^{*}$ \\ ${ }^{1}$ Programa de pós-graduação - Universidade Federal do Vale do São Francisco - Petrolina, PE \\ ${ }^{2}$ Universidade Federal do Vale do São Francisco - Petrolina, PE \\ ${ }^{3}$ Universidade Federal do Vale do São Francisco - Juazeiro, BA
}

\begin{abstract}
RESUMO
Objetivou-se com este trabalho analisar a resistência de placas ósseas de acrílico, confeccionadas com cortadora a laser $\mathrm{CO}_{2}$, quando submetidas às forças que agem sobre o foco de fratura. Foram utilizados 40 fêmures de ovinos (Ovis aries) e confeccionadas 40 placas de acrílico, os quais foram avaliados em grupos: grupo OP - foram avaliados 20 ossos fraturados reduzidos com 20 placas de acrílico; grupo PS foram avaliadas 20 placas de acrílico; e grupo OS - foram avaliados 20 ossos íntegros e frescos. Todos foram submetidos a ensaios mecânicos de compressão, flexão, torção e tração, em máquina de ensaios universal EMIC ${ }^{\circledR} 10000$. Os testes eram interrompidos quando havia fratura do corpo de prova. O grupo PS suportou carga de compressão significativamente superior aos demais grupos OP e OS $(13.255 \pm 290 \mathrm{~N}$, $4.932 \pm 827 \mathrm{~N}$ e $8.681 \pm 303 \mathrm{~N}$, respectivamente). Já em relação à prova de flexão, o grupo OS foi significativamente mais resistente, com média de $2.698 \pm 305 \mathrm{~N}$, em relação aos grupos OP e PS, que obtiveram $1.315 \pm 92 \mathrm{~N}$ e $1.537 \pm 37 \mathrm{~N}$, respectivamente. Conclui-se que as placas de acrílico usinadas a laser apresentam resistência mecânica inferior ao fêmur de ovinos, entretanto, devido à resistência exibida nos testes, demonstraram potencial para serem implantadas em ossos com menor demanda de carga.
\end{abstract}

Palavras-chave: PMMA, osteorredução, biomaterial, biomecânica

\begin{abstract}
The objective of this work was to analyze the resistance of acrylic bone plates, made with $\mathrm{CO}_{2}$ laser cutter, when subjected to the forces acting on the fracture focus. Forty ovine femurs (Ovis aries) were used and 40 acrylic plates were prepared and evaluated in groups: In the OP Group, 20 fractured bones reduced with 20 acrylic plates were evaluated; in the PS Group, 20 acrylic plates were evaluated; and in the OS Group 20 intact and fresh bones were evaluated. All were submitted to mechanical tests of compression, bending, twisting and traction in an EMIC ${ }^{1} 10000$ universal testing machine. The tests were interrupted when the body of proof was fractured. The PS group supported a significantly higher compression load than the other OP and OS groups $(13,255 \pm 290 \mathrm{~N}, 4,932 \pm 827 \mathrm{~N}$ and 8,681 $\pm 303 \mathrm{~N}$, respectively). Regarding the flexural test, the OS group was significantly more resistant, with a mean of $2,698 \pm 305 N$, compared to the groups $O P$ and $P S$ that obtained $1,315 \pm 92 N$ and $1,537 \pm 37 N$ respectively. It is concluded that the laser-machined acrylic plates have inferior mechanical resistance to the femur of the ovine, however, due to the presented resistance, it shows potential to be implanted in bones with lower load demand.
\end{abstract}

Keywords: PMMA, osteoreduction, biomaterial, biomechanics

Recebido em 28 de agosto de 2017

Aceito em 15 de abril de 2019

* Autor para correspondência (corresponding author)

E-mail: alexandre.antonelli@univasf.edu.br 


\section{INTRODUÇÃO}

As lesões ósseas representam um sério problema na medicina veterinária. $\mathrm{O}$ aumento de sua apresentação na rotina clínica cirúrgica e as complicações inerentes à reparação óssea têm intensificado a busca por biomateriais que possam, ao mesmo tempo, mimetizar, o máximo possível, o tecido afetado, assim como suportar as exigências mecânicas da função óssea, pois o paciente veterinário não possui consciência da necessidade de repouso do membro em recuperação. $\mathrm{O}$ biomaterial deve também proporcionar boa biocompatibilidade, apresentar facilidade de produção e utilização, e sobretudo baixo custo (Moraes et al., 2007; Burguer et al., 2013; Ratner et al., 2013; Mahyudin e Hermawan, 2016).

O objetivo da reparação óssea, quando da utilização de implantes ortopédicos, é reduzir a movimentação do foco de fratura e eliminar, se possível, as forças de compressão, torção, tração, flexão e cisalhamento, que agem diretamente sobre os fragmentos fraturados, transferindo a força do foco de fratura para a placa. Diversos biomateriais podem ser utilizados como implantes ortopédicos, tais como ligas metálicas, polímeros e biovidros, sendo as ligas de aço inoxidável e o polímero polimetilmetacrilato amplamente utilizados como matéria-prima de implantes veterinários (Dantas et al., 2011; Deshmukh e Kulkarni, 2015; Cattelo et al., 2017).

A liga metálica é representada pelo aço inox ASTM F 138 ou AISI 316L, que é a liga amplamente utilizada em implantes na medicina veterinária (Jaimes et al., 2008; Burguer et al., 2013; Drago et al., 2015). Contudo, apesar de suas excelentes propriedades mecânicas de suporte e transferência de carga, sua utilização é indicada para implantes temporários, e seus efeitos deletérios, como rejeição exacerbada, metalose, risco de desenvolvimento de neoplasias no osso adjacente, além do risco de refraturas na retirada do implante, contribuem negativamente para sua maciça utilização (Drago et al., 2015).

O PMMA ou polimetilmetacrilato é um polímero amorfo, inerte, utilizado há mais de 60 anos como cimento ósseo e resina para odontologia, com excelentes resultados (Dantas et al., 2011;
Burguer et al., 2013), semelhantes aos resultados obtidos por Cardona et al. (2011), que produziram implantes para defeitos cranianos com impressora 3D com excelente implantação e baixo custo relativo. Contudo, no processo de polimerização, existem alguns fatores que limitam seu uso, tais como rendimento de reação química instável, que permite a liberação de monômeros de metilmetacrilato livres, bem como altas temperaturas durante o processo de polimerização (Roehsig et al., 2008; Dantas et al., 2011; Burguer et al., 2013).

Para evitar esses fatores limitantes, uma alternativa seria a utilização do PMMA já polimerizado, puro, comercializado em chapas, para fabricação de placas ósseas, devido a sua alta usinabilidade e baixo custo. Todavia, não há relatos na literatura da utilização desse material como base para a fabricação de placas ósseas, demandando testes mecânicos que simulem as forças que agem sob o foco de fratura para conhecer o comportamento mecânico da placa de PMMA e elencá-la como um possível biomaterial para implantes. Portanto, com este trabalho, objetivou-se analisar a resistência mecânica de placas ósseas de acrílico.

\section{MATERIAL E MÉTODOS}

De acordo com a Comissão de Ética no Uso de Animais (Ceua) da Universidade Federal do Vale do São Francisco (Univasf), segundo as orientações do Conselho Nacional de Controle de Experimentação Animal (Concea), pesquisas científicas que não utilizam animais vivos em nenhuma fase de seu desenvolvimento não necessitam de certificação de uso ético de animais. Entende-se por utilização de animais: manipulação, captura, coleta, criação, experimentação (invasiva ou não invasiva), realização de exames ou procedimentos cirúrgicos, ou qualquer outro tipo de intervenção que possa causar estresse, dor, sofrimento, mutilação e/ou morte. Como o presente trabalho não realizou nenhuma das atividades supracitadas e todas as peças utilizadas foram adquiridas em local de comercialização de carne, onde elas seriam descartadas, não houve a necessidade de certificação pelo Ceua-Univasf.

Foram utilizados 40 fêmures frescos de ovino (Ovis aires), obtidos em estabelecimento comercial especializado em carne ovina, na 
cidade de Petrolina, PE. Os ossos tinham, em média, $25 \mathrm{~cm}$ de comprimento total e diâmetro ósseo médio de $5 \mathrm{~cm}$ na diáfise, com cortical média de $6 \mathrm{~mm}$, mensuradas por exames radiográficos executados no Laboratório de Diagnóstico por Imagem Veterinária (Labdive), Setor de Radiologia, do Hospital Veterinário Universitário (HVU) da Univasf.

Foram confeccionadas 40 placas de neutralização, feitas de chapas PMMA cast puras, adquiridas na Acriplanos Distribuidora de
Acrílicos. As placas foram usinadas com auxílio de uma máquina de corte a laser $\mathrm{CO}_{2}$, modelo DS1312, fabricada pela Ds $4{ }^{\circledR}$ Laser - São Paulo, com velocidade de corte de $10 \mathrm{~mm} / \mathrm{s}$ e com potência de laser de 35\%, para que houvesse secção total do acrílico e bordas polidas. As placas tinham $10 \mathrm{~cm}$ de comprimento por $8 \mathrm{~mm}$ de largura e $10 \mathrm{~mm}$ de altura; cada placa tinha seis furos com $2,7 \mathrm{~mm}$ de diâmetro total para passagem dos parafusos de aço inox, como visto na Fig. 1.

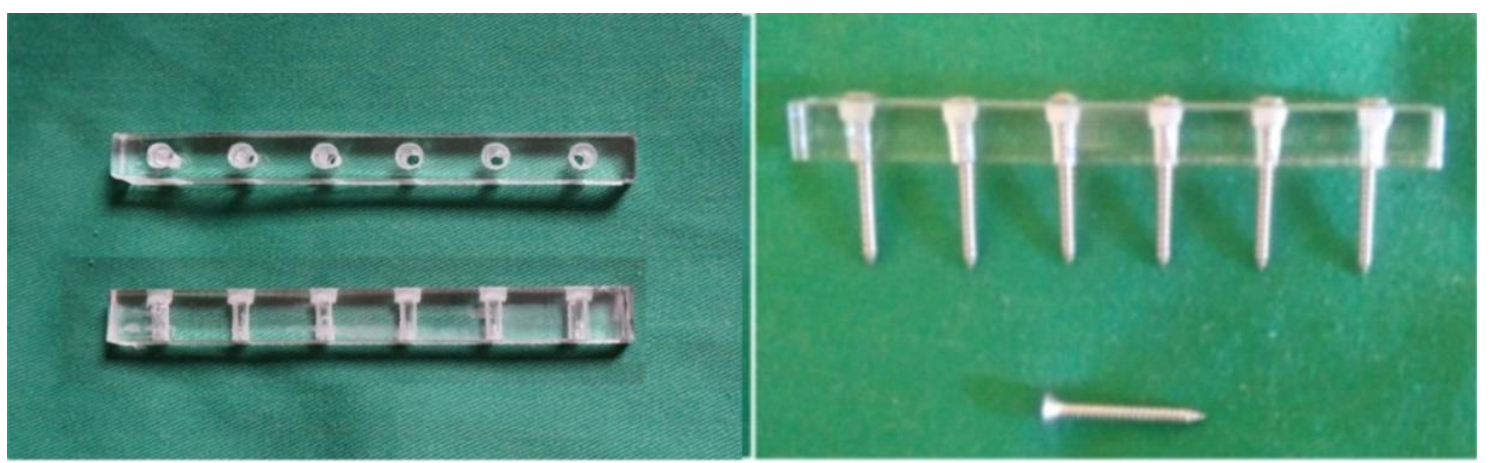

Figura 1. Placas de PMMA com $10 \mathrm{~cm}$ de comprimento por $8 \mathrm{~mm}$ de largura e $10 \mathrm{~mm}$ altura e seis furos de $2,7 \mathrm{~mm}$ de diâmetro, confeccionadas a laser e utilizadas neste experimento.

Os fêmures frescos foram limpos, sem sofrerem nenhum tipo de procedimento de maceração, na tentativa de se reproduzirem ao máximo as condições ósseas naturais. Após a limpeza, todos eles foram cimentados em bases de PVC. Foram feitos furos nos ossos com uma broca de aço rápido de $3,7 \mathrm{~mm}$, e esses foram fixados pelos orifícios na base de PVC com os parafusos de $3,7 \mathrm{~mm}$ de espessura fabricados em aço inox 316L. Após serem fixadas nas bases de PVC, as amostras de fêmures ovinos foram fraturadas com auxílio de uma serra manual $\left(\right.$ Scarrett $^{\circledR}$ $3 \mathrm{M}^{\mathrm{TM}}$ ), de $3 \mathrm{~mm}$ de espessura, de modo a simular uma fratura transversa, gerando dois fragmentos ósseos. Depois de realizadas as osteoclases, todas as placas de neutralização foram fixadas na face lateral do fêmur, seguindo as recomendações da AOVET, utilizando-se parafusos de aço inox de 2,7mm, os quais foram parafusados com auxílio de uma parafusadeira portátil de 3000 rpm $\left(\right.$ Skill $^{\circledR}$, Bosch $\left.^{\mathrm{TM}}\right)$, sem análise de torque, sendo apenas observado o travamento do parafuso nas duas corticais e o contato das placas de PMMA com o tecido ósseo a olho nu.

Todos os testes mecânicos foram realizados no Laboratório de Ensaios Materiais da
Universidade Federal do Vale do São Francisco, Campus Juazeiro, em Juazeiro, BA. Foi utilizada a máquina de ensaios universal (modelo DL 10000 - EMIC $^{\mathrm{TM}}$, Instron Brasil, Paraná), com capacidade de $100 \mathrm{kN}$ e células de carga de $2 \mathrm{t}$, acoplada a um desktop para que os dados fossem analisados e gerados pelo software TESC, previamente instalado.

Os ensaios mecânicos foram realizados em três grupos distintos, sendo eles:

grupo osso-placa (OP) - constituído por 20 conjuntos de placa de PMMA fixada a osso fraturado com auxílio de parafusos de aço inox; grupo placa somente (PS) - constituído apenas por 20 placas de PMMA;

grupo osso somente (OS) - constituído apenas por 20 ossos íntegros sem fraturas.

Todos os grupos foram submetidos aos mesmos ensaios mecânicos de compressão e flexão, a fím de se comparar o desempenho mecânico de cada corpo de prova sob as mesmas condições, e apenas os grupos OP e OS foram submetidos aos ensaios de tração e torção. Para todos os testes, foi considerada falha o momento em que ocorria a ruptura do corpo de prova. $\mathrm{O}$ ensaio mecânico 
de compressão teve como objetivo comprimir o corpo de prova, a fim de avaliar o suporte à carga e à deformação do conjunto. Para esse ensaio, foram estabelecidos, como parâmetro de précarga, valores que variaram de $5-10 \mathrm{~N}$ para fixação no equipamento, com velocidade no ensaio de $5 \mathrm{~mm} / \mathrm{min}$, com célula de carga de $2 \mathrm{t}$ (Fig. 2A). No ensaio de flexão de três pontos, o corpo de prova foi posicionado sobre dois roletes de suporte na mesa do equipamento de ensaios, com separação de $50 \mathrm{~mm}$ entre as bases. $\mathrm{Na}$ secção móvel do equipamento, foi utilizada célula de carga de $2 \mathrm{t}$, sendo a pré-carga estabelecida de $5-10 \mathrm{~N}$ e a velocidade de $5 \mathrm{~mm} / \mathrm{mim}$ (Fig. 2B).
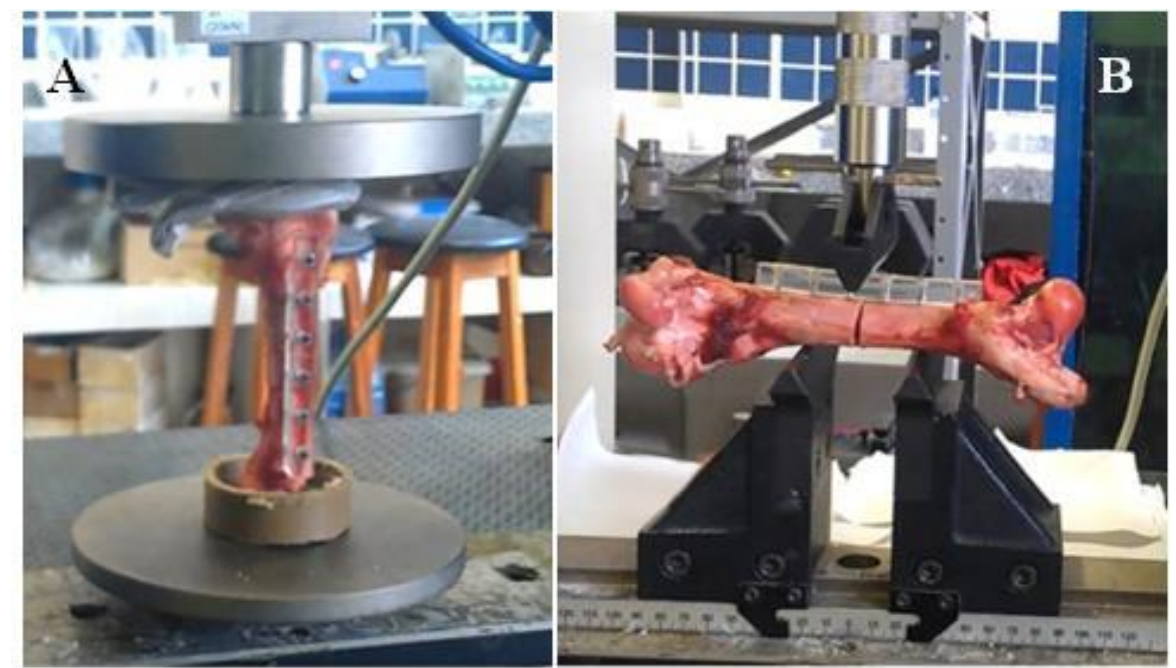

Figura 2. Fêmur de ovino (Ovis aries) fraturado e estabilizado com placa de PMMA, acoplado à máquina de ensaios universal EMIC DL, durante ensaio mecânico de compressão (A) e durante ensaio de flexão de três pontos $(\mathrm{B})$.

No ensaio de tração, o corpo foi tracionado em vetores opostos até a ruptura do corpo de prova. Os parâmetros de pré-carga de tração, velocidade de tração e célula de carga foram semelhantes aos utilizados nos testes de compressão (Fig. 3A). Para os ensaios de torção, foi utilizado o dispositivo desenvolvido por Cardoso e Oliver (2019), que permite a realização desse tipo de ensaio com a máquina de ensaios universal. Os parâmetros de configuração do teste foram semelhantes ao outros ensaios de compressão e tração (Fig. 3B).

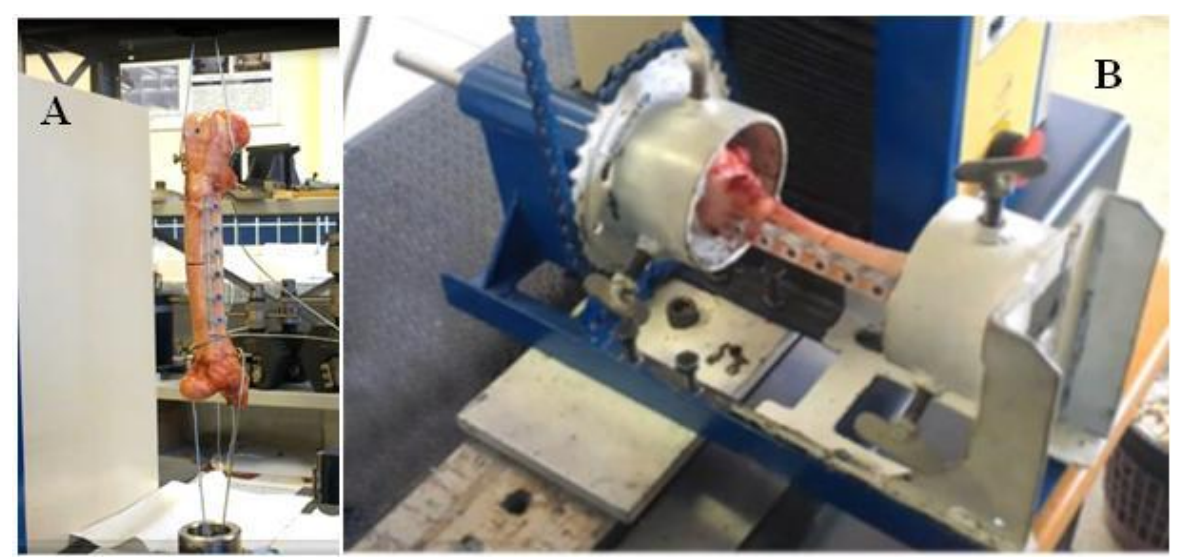

Figura 3. Fêmur de ovino (Ovis aries) fraturado e estabilizado com placa de PMMA, acoplado à máquina de ensaios universal EMIC DL, durante ensaio de tração (A) e durante ensaio de torção (B). 
Para se avaliarem os resultados dos testes de compressão, tração e flexão, fez-se necessário transformar as medidas de Newtons (N) para quilograma-força (kgf) ou megapascal (MPa). Para os testes de torção, foi necessário conhecer o momento torsor e o ângulo de torção, que mostra exatamente o ângulo máximo suportado na torção pelo implante de PMMA (Sod et al., 2006). O delineamento utilizado foi blocos ao acaso, com três tratamentos e cinco repetições. Foi realizado $o$ teste de normalidade Kolmogorov-Smirnov, e, em seguida, foi feita comparação de médias entre os dados dos grupos OP e OS, e entre os grupos OS e PS, utilizandose teste $\mathrm{t}$ de Student. Foram consideradas significativas as diferenças cujo valor de $\mathrm{P}$ apresentou valores iguais ou inferiores a 0,05 .

\section{RESULTADOS E DISCUSSÃO}

Segundo Hammel et al. (2006), a utilização de testes biomecânicos apresenta resultados confiáveis por simular as forças in vivo que atuam em placas ósseas em focos de fraturas estabilizadas antes de sua recuperação. Também se optou por utilizar ossos frescos, e não modelos sintéticos, pois, segundo Gordon et al. (2010), modelos sintéticos não apresentam as mesmas características biomecânicas dos ossos, apesar de minimizarem a variação em relação à mineralização e à porosidade dos ossos de diferentes indivíduos. Em relação às fraturas, optou-se por osso com osteotomia, com o objetivo de simular uma fratura típica sem fragmentos, sem distração, mantendo o tamanho fisiológico do osso, tal como proposto por Bernarde et al. (2001).

Neste estudo, os ensaios biomecânicos foram realizados sob condições normatizadas, em que os corpos de prova tinham dimensões padronizadas, preservando posicionamentos idênticos para cada teste, como proposto por Drago et al. (2015), que citam que as propriedades mecânicas do osso sofrem influência do sentido das fibras colágenas e da secção do osso a ser analisada. Os corpos de prova foram cimentados em suas extremidades, com a finalidade de estarem perfeitamente acoplados ao equipamento, de modo que não houvesse movimentação da peça em sentido diferente da compressão axial. Tal metodologia mostrou-se adequada, pois não houve deslocamento de nenhum corpo de prova durante a realização dos testes.

As placas de neutralização de PMMA criadas com $100 \mathrm{~mm}$ de comprimento por $8 \mathrm{~mm}$ de largura e $10 \mathrm{~mm}$ de espessura e seis furos são semelhantes a uma placa comercial de aço cirúrgico ASTM F 138, que apresenta $103 \mathrm{~mm}$ de comprimento por $12 \mathrm{~mm}$ de largura e $4 \mathrm{~mm}$ de espessura e seis furos. Uma clara vantagem das placas de PMMA é o peso médio, que foi de $8,4 \mathrm{~g}$, bem inferior ao peso médio das placas de aço cirúrgico de mesmo comprimento e largura que é de $44 \mathrm{~g}$. Entretanto, a espessura das placas de PMMA foi maior (4-6mm), o que poderia dificultar o fechamento da ferida cirúrgica e a posição correta do tecido ósseo adjacente, podendo prejudicar a deambulação do paciente, dificultando a sua aplicação clínica. Mas, para se analisar essa suposição, faz-se necessária a realização de testes in vivo para avaliação desse parâmetro.

Ao se analisarem os resultados de força máxima suportada nos ensaios mecânicos, nos testes de compressão, comparando-se o grupo osso somente (OS) com o grupo osso com placa (OP), houve uma redução significativa de $44 \%$ no suporte à força máxima de compressão no grupo OP, conforme apresentado na Tab. 1.

Contudo, quando analisada a tensão máxima de suporte na compressão, o grupo $\mathrm{OP}$ apresentou desempenho superior, com um aumento de quase $650 \%$ quando comparado com o grupo OS. Esse aumento significativo pode ser devido à combinação dos elementos placa, parafuso e osso, que podem ter interferido diretamente no suporte à carga, além da possibilidade de esse baixo desempenho do grupo OS estar relacionado à área total da cortical óssea que é submetida à pressão, a qual, neste estudo, foi de $829 \mathrm{~mm}^{2}$ em comparação com $73 \mathrm{~mm}^{2}$ da placa de PMMA. De acordo com Cardoso e Oliver (2019), quanto menor a área de secção, maior será a pressão exercida sobre ela, justificando também a queda de desempenho do grupo OS quando comparado com o grupo OS nesse parâmetro. 
Tabela 1. Valores médios e erro-padrão da média das forças máximas ( $\mathrm{N}$ e kgf), da tensão máxima (MPa) e da deformação $(\mathrm{mm})$ apresentadas por fêmures ovinos com ou sem placa de neutralização de PMMA e pelas placas de PMMA, submetidos a testes de compressão e flexão

\begin{tabular}{|c|c|c|c|c|c|c|}
\hline \multirow{2}{*}{$\begin{array}{l}\text { Testes } \\
\text { mecânicos }\end{array}$} & \multirow[b]{2}{*}{ Medições } & \multicolumn{3}{|l|}{ Grupos } & \multirow{2}{*}{$\begin{array}{l}\text { OP } \\
\mathrm{x} \\
\text { OS }\end{array}$} & \multirow{2}{*}{$\begin{array}{l}\text { OS } \\
\mathrm{x} \\
\mathrm{PS}\end{array}$} \\
\hline & & OP & OS & PS & & \\
\hline \multirow{4}{*}{ Compressão } & Força máxima $(\mathrm{N})$ & $4932 \pm 827$ & $8681 \pm 303$ & $13255 \pm 290$ & $\leq 0,05$ & $\leq 0,05$ \\
\hline & Força (kgf) & $502 \pm 84,3$ & $884 \pm 30,9$ & $1351 \pm 29,3$ & $\leq 0,05$ & $\leq 0,05$ \\
\hline & $\begin{array}{l}\text { Tensão máxima } \\
(\mathrm{MPa})\end{array}$ & $67,7 \pm 11,3$ & $10,4 \pm 14,8$ & $151,5 \pm 14,8$ & $\leq 0,05$ & $\leq 0,05$ \\
\hline & Deformação (mm) & $5,19 \pm 0,31$ & $2,99 \pm 0,45$ & $4,27 \pm 0,39$ & $\leq 0,05$ & n.s. \\
\hline \multirow{4}{*}{ Flexão } & Força máxima $(\mathrm{N})$ & $1315 \pm 92,0$ & $2698 \pm 305$ & $1537 \pm 37,0$ & $\leq 0,05$ & $\leq 0,05$ \\
\hline & Força (kgf) & $134 \pm 9,7$ & $275 \pm 31,1$ & $156 \pm 3,7$ & $\leq 0,05$ & $\leq 0,05$ \\
\hline & $\begin{array}{l}\text { Tensão máxima } \\
(\mathrm{MPa})\end{array}$ & $18,0 \pm 1,30$ & $3,3 \pm 0,40$ & $19,2 \pm 0,50$ & $\leq 0,05$ & $\leq 0,05$ \\
\hline & Deformação $(\mathrm{mm})$ & $2,05 \pm 0,14$ & $2,99 \pm 0,45$ & $2,92 \pm 0,22$ & $\leq 0,05$ & n.s. \\
\hline
\end{tabular}

Tais resultados de força máxima se mostram superiores aos encontrados por Drago et al. (2015), que, ao usarem placas derivadas de osso bovino, obtiveram redução de $80 \%$ na tensão máxima após serem implantadas em tíbias de coelho. Também foram muito superiores aos resultados das placas de compressão dinâmica, fabricadas por meio da moldagem de PMMA autopolimerizável, obtidos por Cardona et al. (2011) e Catello et al. (2017), que obtiveram $210 \mathrm{~N}$ nos ensaios de compressão da placa de cimento ósseo e $1957 \mathrm{~N}$ com cimento ósseo, respectivamente, em comparação a uma força máxima média de $4932 \mathrm{~N}$ da placa de PMMA confeccionada a laser no presente estudo.

As placas ósseas de PMMA usinadas a laser a partir de placas de PMMA industrializadas, diferentemente das placas ósseas de PMMA moldadas utilizadas por Cardona et al. (2011) e Catello et al. (2017), apresentaram estrutura polimerizada totalmente uniforme, sem a presença de bolhas ou rugosidades. Para os ensaios de flexão de três pontos, não houve a necessidade de fixação dos corpos de prova na base de PVC, pois não era necessário o uso de garras para apreensão de nenhum dos corpos de prova ensaiados, e o posicionamento correto do osso na máquina de ensaios universal nesse teste era facilmente obtido, como proposto por Sod et al. (2006), Drago et al. (2015), e Goes Filho (2016)

Nos ensaios de flexão, o grupo OS apresentou resistência significativamente superior em comparação aos grupos OP e OS, conforme apresentado na Tab. 1. Tal resultado era esperado devido à natureza frágil do PMMA, que, por se tratar de um polímero, tem comportamento mecânico mais frágil (Ratner et al., 2013), evidenciado pelo fato de o local da fratura do implante ser sempre próximo aos furos e com características de fratura diagonal do implante, semelhante ao obtido por Mataliotakis et al. (2015), que utilizaram placas moldadas de PMMA.

Os resultados de flexão obtidos no presente trabalho, no entanto, superam os alcançados por Cardona et al. (2011), que, nos testes de flexão de placas de PMMA autopolimerizável, obtiveram $210 \mathrm{~N}$, resultado que corresponde a $13,34 \%$ do valor médio atingido pelo grupo PS, que foi de $1537 \mathrm{~N}$. Entretanto, se comparado com placas de metal, como realizado por Sod et al. (2006), o desempenho é muito inferior, uma vez que os referidos autores obtiveram o valor médio de $191.100 \mathrm{~N}$ no ensaio de flexão.

Quanto às deformações nos ensaios de compressão e flexão, pode-se observar que o grupo OP apresentou deformação significativamente superior ao grupo OS (Tab. 1) nos ensaios de compressão, devido à presença dos parafusos que auxiliam na dissipação da força, melhorando a distribuição da carga e, assim, favorecendo o comportamento elástico do PMMA (Cardoso e Oliver, 2019). Quando comparado com o grupo PS, o grupo OS não apresentou diferença significativa, em razão de a resistência e os módulos de elasticidade do acrílico e do osso serem muito semelhantes (Cardona et al., 2011; Ratner et al., 2013). 
Os resultados de deformação obtidos no presente estudo $(4,27 \mathrm{~mm}$ e $2,99 \mathrm{~mm}$, para acrílico e osso, respectivamente) são superiores aos alcançados por Cattelo et al. (2017), que mostraram deformação média de $1,04 \mathrm{~mm}$ e $0,52 \mathrm{~mm}$ para acrílico e osso, respectivamente. Essa diferença se fez presente pela natureza da amostra utilizada como corpo de prova, pois, no estudo de Cattelo et al. (2017), foram empregados pequenos cilindros de acrílico e osso cortical, diferindo em mais de $300 \%$ da placa de PMMA e do osso íntegro no presente trabalho.

Nos ensaios de flexão, o comportamento de deformação do grupo OP foi significativamente inferior ao grupo OS, com valores médios de deformação de 2,05mm e 2,94mm, respectivamente (Tab. 1). Essa queda de desempenho está relacionada à presença dos furos, que diminuem a resistência mecânica por diminuírem a área sob ação da força aplicada na placa, que, no caso deste ensaio, independem da presença do parafuso. Quando comparados com os resultados obtidos por Sod et al. (2006), que utilizaram placas metálicas, a deformação alcançada foi inferior à do presente estudo, $2,05 \mathrm{~mm}$ do PMMA contra $1,8 \mathrm{~mm}$ do aço inox, fato esse que decorre de a resistência mecânica do metal ser bastante superior à do PMMA, com módulos de elasticidade totalmente distintos
(Mahyuidin e Hermawan, 2016). Essa menor deformação é indesejada do ponto de vista da consolidação, pois as placas de acrílico, por permitirem maior movimentação dos fragmentos ósseos, estimulam, de forma mais eficiente, a cicatrização, além de apresentarem características elásticas semelhantes ao osso, mimetizando, com maior eficiência, o tecido ao qual são implantadas, característica essa desejada para biomateriais (Bankoff, 2007; Williams, 2009; Fossum, 2014; Fernandes et al., 2015).

Não foi possível realizar os testes de tração e torção nos corpos de prova do grupo OS, devido à impossibilidade de se fixar o corpo de prova na máquina de ensaios universal para obtenção dos dados. O cimento ósseo utilizado para fixar o corpo de prova fraturava-se antes do osso. Tentou-se o uso de cabos de aço para ancoragem do osso, mas a deformação era prejudicada pela ação do desses cabos. Assim, foram realizados os testes de tração e torção apenas com os grupos OP e PS. Nos ensaios de tração, os resultados obtidos para o grupo $O P$ foram significativamente inferiores aos alcançados com o grupo PS, em que é possível verificar uma queda significativa de quase $80 \%$ na resistência à força máxima de tração axial, conforme apresentado na Tab. 2.

Tabela 2. Valores médios e erro-padrão da média da força máxima $(\mathrm{N})$, da força (kgf), da pressão máxima $(\mathrm{MPa})$ e da deformação $(\mathrm{mm})$, nos testes de tração, e da força máxima $(\mathrm{N})$, dos momentos angulares da força, do ângulo de torção (Rad) e do ângulo máximo de torção $\left(^{\circ}\right)$, apresentados por fêmures ovinos com placa de neutralização de PMMA e pelas placas de PMMA, submetidos a testes de torção

\begin{tabular}{|c|c|c|c|}
\hline \multirow{2}{*}{$\begin{array}{c}\text { Testes } \\
\text { mecânicos }\end{array}$} & \multirow{2}{*}{ Medições } & \multicolumn{2}{|c|}{ Grupos } \\
\hline & & OP & PS \\
\hline \multirow{4}{*}{ Tração } & Força máxima $(\mathrm{N})$ & $452 \pm 43,0^{b}$ & $2389 \pm 292^{\mathrm{a}}$ \\
\hline & Força (kgf) & $46,1 \pm 4,4^{\mathrm{b}}$ & $243 \pm 29,8^{a}$ \\
\hline & Tensão máxima (MPa) & $6,2 \pm 0,6^{\mathrm{b}}$ & $29,9 \pm 3,7^{\mathrm{a}}$ \\
\hline & Deformação (mm) & $20,85 \pm 2,03^{\mathrm{a}}$ & $1,06 \pm 0,15^{b}$ \\
\hline \multirow{5}{*}{ Torção } & Força máxima $(\mathrm{N})$ & $106,1 \pm 9,8$ & $102,6 \pm 4,3$ \\
\hline & Momento angular (N.m²) & $7,4 \pm 0,7$ & $7,2 \pm 0,3$ \\
\hline & Ângulo de torção - Tg $\theta$ (Rad) & $0,35 \pm 0,03^{\mathrm{a}}$ & $0,18 \pm 0,02^{b}$ \\
\hline & Ângulo máximo $\left(^{\circ}\right)$ & $19,0 \pm 1,5^{\mathrm{a}}$ & $10,1 \pm 0,9^{b}$ \\
\hline & Deformação (mm) & $24,4 \pm 2,12^{\mathrm{a}}$ & $12,74 \pm 1,09^{b}$ \\
\hline
\end{tabular}

${ }^{\mathrm{a}, \mathrm{b}}$ Letras distintas nas linhas indicam diferença significativa entre os grupos $(\mathrm{P} \leq 0,05)$.

Nos ensaios de torção, os grupos OP e PS se comportaram de forma muito semelhante, devido ao fato de a força utilizada no momento torsor trabalhar totalmente sobre a placa de PMMA e, assim, o osso e os parafusos não influenciarem na força aplicada. Contudo, a angulação obtida até a quebra pelo grupo $\mathrm{OP}$ foi significativamente maior em $80 \%$ que o valor alcançado pelo grupo PS (Tab. 2). Quando comparadas com os resultados obtidos por Cardona et al. (2011), que utilizaram placa de PMMA autopolimerizável, as placas ósseas de 
PMMA confeccionadas a laser neste estudo apresentaram desempenho $257 \%$ superior $\left(2,8 \mathrm{~N} / \mathrm{m}^{2}\right.$ e $7,2 \mathrm{~N} / \mathrm{m}^{2}$, respectivamente).

Apesar da impossibilidade de se obterem os dados de tração e torção do grupo OS e de não se realizar a análise estatística nesses ensaios para compararação com os demais grupos, a maioria das normas técnicas consideram as forças de compressão e flexão como testes fidedignos para avaliação do comportamento mecânico de um implante (Drago et al., 2015; Catello et al., 2017; Goes Filho, 2016). Segundo Mataliotakis et al. (2015), placas de PMMA são mais vantajosas, pois possuem a mesma aplicação de outras placas, não permitindo nenhum contato do osso com o ambiente, além de não serem totalmente radiopacas, o que permite o acompanhamento radiográfico da cicatrização e da regeneração óssea no interior da fratura. A placa de PMMA usinada a laser pode também ser fabricada sob medida, de acordo com o tamanho do animal, com a fratura, ou com as necessidades biomecânicas do paciente. O PMMA é um material que pode ser impregnado com antibiótico, utilizando-se sistema de liberação de fármaco local, como o realizado por McKee et al. (2010), que avaliaram o uso de antibiótico impregnado em PMMA depois de debridamento cirúrgico em pacientes com osteomielite crônica.

\section{CONCLUSÕES}

Pode-se concluir que as placas de PMMA fabricadas a partir de chapas cast de PMMA puro, implantadas ao fêmur de ovino fraturado, têm desempenho mecânico nos ensaios de compressão e flexão inferior ao osso íntegro, mas, quando isoladas, apresentam resistência mecânica superior ao tecido ósseo. De acordo com os resultados dos testes mecânicos, as placas de PMMA usinadas podem ser uma opção viável em pacientes veterinários com fraturas em ossos com menor demanda de suporte mecânico, tanto por sua característica como biomaterial quanto pelo baixo custo, aliado à facilidade de produção e customização, o que leva à necessidade de novos trabalhos que avaliem seu comportamento in vivo para um melhor entendimento do comportamento mecânico e biológico dessas placas.

\section{AGRADECIMENTOS}

O presente trabalho foi realizado com apoio da Coordenação de Aperfeiçoamento de Pessoal de Nível Superior (Capes), Brasil, código de financiamento 001 .

\section{REFERÊNCIAS}

BANKOFF, A.D.P. Morfologia e cinesiologia aplicada ao movimento humano. Rio de Janeiro: Guanabara Koogan, 2007. 328p.

BERNARDE, A.; DIOP, A.; MAUREL, N.; VIGUIER, E. An in vitro biomechanical study of bon plate and interlocking nail in a canine diaphyseal femoral model. Vet. Surg., v.30, p.397-408, 2001.

BURGUER, C.P.; MORAES, P.C.; MANISCALCO, C.L. et al. Cimento de aluminato de cálcio: uso em defeitos ósseos induzidos em fêmur de coelhos. Arq. Bras. Med. Vet. Zootec., v.65, p.757-762, 2013.

CARDONA, L.; BROUSSE, M.; MIERES, M. et al. Evaluación de la resistencia de un prototipo de placa de compresión dinámica (PCD) fabricada de polimetilmetacrilato (PMMA) probada en fémur canino osteotomizado. Rev. Med. Vet., v.21, p.13-24, 2011.

CARDOSO, P.H.N.; OLIVIER, N.C. Projeto $e$ fabricação de um dispositivo de ensaio de torção: uma maneira inovadora e de baixo custo para realização de ensaios de torção. Riga: Novas Edições Acadêmicas, 2019. 104p.

CATELLO, J.D.C.; DÓRIA, R.G.S.; FANTINATO NETO, P. et al. Estudo comparativo da resistência mecânica da força de compressão entre biomateriais naturais, sintéticos e mistos. Pesqui. Vet. Bras., v.37, p.91-96, 2017.

DANTAS, T.S.; LELIS, E.R.; NAVES, L.Z. et al. Materiais de enxerto ósseo e suas aplicações na odontologia. J. Health Sci., v.13, p.131-135, 2011.

DESHMUKH, R.M.; KULKARNI, S.S. A review on biomaterials in orthopedic bone plate application. Int. J. Curr. Eng. Technol., v.5, p.2587-2591, 2015. 
DRAGO, M.A.; DRAGO, M.; CERQUEIRA, H.D.B. et al. Avaliação ex vivo das propriedades mecânicas em flexão de placas ósseas bovina na osteossíntese de tíbias de coelhos. Rev. Bras. Med. Vet., v.37, p.245-249, 2015.

FERNANDES, M.G.A.; FONSECA, E.M.; BARBOSA, M.P.; NATAL, R.M. Análise biomecânica das fraturas diafisárias do fémur (Tipo B) utilizando uma haste intramedular. Rev. Iberoam. Ing. Mec., v.19, p.41-48, 2015.

FOSSUM, T. Cirurgia de pequenos animais. 4.ed. Rio de Janeiro: Elsevier. 2014. 1619p.

GOES FILHO, P.R. Confecção e avaliação mecânica de implantes ortopédicos produzidos em poli (L-ácido lático)(PLLA) por impressoras 3D. 2016. 54f. Dissertação (Mestrado) Universidade Federal Rural de Pernambuco, Recife, PE.

GORDON, S.; MOENS, N.M.M.; RUNCIMAN, J.; MONTEITH, G. The effect of the combination of locking screws and non-locking screws on the torsional properties of a lockingplate construct. Vet. Comp. Orthop., v.23, p.7-13, 2010.

HAMMEL, S.P.; PLUHAR, G.E.; NOVO, R.E. et al. Fatigue analysis of plates used for fracture stabilization in small dogs and cats. Vet. Surg., v.35, p.573-578, 2006.

JAIMES, R.F.V.; AFONSO, M.L.C.A.; ROGERO, S.O. et al. Novos aços inoxidáveis aplicados a implantes ortopédicos: estudo eletroquímico dos aços P558 isento de níquel e do aço UNS S31254 em meio físiológico. In: CONGRESO DE LA SOCIEDAD IBEROAMERICANA DE ELECTROQUÍMICA, 17., 2008, Medellín. Anais... Medellín: SIBAE, 2008. p.1-7.

MAHYUDIN, F.; HERMAWAN, H. Biomaterials and medical devices: a perspective from an emerging country. Genebra: Springer, 2016. v.582, 42p.
MATALIOTAKIS, G.I.; TSOUKNIDAS, A.; PANTELIOU, S. et al. A new, low cost, locking plate for the long-term fixation of a critical size bone defect in the ratfemur: in vivo performance, biomechanical and finite element analysis. Biomed. Mater. Eng., v.25, p.335-346, 2015.

MCKEE, M.D.; LI-BLAND, E.A.; WILD, L.M.; SCHEMITSCH, E.H. A prospective, randomized clinical trial comparing an antibioticimpregnated bioabsorbable bone substitute with standard antibiotic-impregnated cement beads in the treatment of chronic osteomyelitis and infected nonunion. J. Orthop. Trauma, v.24, p.483-490, 2010.

MORAES, P.C.; PADILHA FILHO, J.G.; CANOLA, J.C. et al. Uso do ultra-som de baixa intensidade em falhas ósseas produzidas experimentalmente em rádios de coelhos, preenchidas ou não com cimento de fosfato de cálcio. Vet. Notícias, v.13, p.67-74, 2007.

RATNER, B.D.; HOFFMAN, A.S.; SCHOEN, F.J.; LEMONS, J.E. Biomaterials Science: an introduction to materials in medicine. 3.ed. Oxford: Elsevier, 2013. 1573p.

ROEHSIG, C.; ROCHA, L.B.; BARAUNA JUNIOR, D. et al. Fixação de fraturas ilíacas em cães com parafusos, fios de aço e cimento ósseo de polimetilmetacrilato. Cienc. Rural, v.38, p.1675-1681, 2008.

SOD, G.A.; HUBERT, J.D.; MARTIN, G.S.; GILL, M.S. An in vitro biomechanical comparison between prototype tapered shaft cortical bone screws and AO cortical bone screws for an equine metacarpal dynamic compression plate fixation of osteotomized equine third metacarpal bones. Vet. Surg., v.35, p.634-642, 2006.

WILLIAMS, D.F. On the nature of biomaterials. Biomater, v.30, p.5897-5909, 2009. 